\title{
L'intérêt des calculs éléments finis tridimensionnels dans les projets géotechniques
}

\section{A. GUILLOUX}

Terrasol

42-52, quai de la Rapée 75583 Paris Cedex 12
L'article a pour objet de présenter un avis sur l'intérêt des calculs éléments finis tridimensionnels dans les projets géotechniques. Après une revue des avantages, évidents, de ce type de modélisation, mais aussi de ses limites, il présente un certain nombre de cas réels où la méthode a été utilisée, et qui illustrent à la fois ses atouts et ses faiblesses. Il conclut sur certains cas où ce type de modélisations 3D peut être considéré comme d'un usage courant, ne posant pas de difficultés majeures, du fait de la relative simplicité du projet, mais avec un caractère tridimensionnel très marqué. En revanche dans certains projets, souvent plus extrêmes en raison de la complexité de l'ouvrage, la modélisation 3D peut effectivement apporter des réponses à des problèmes auxquels il est impossible de répondre simplement, mais au prix d'efforts importants et souvent d'hypothèses simplificatrices, qui doivent inciter à la prudence quant à l'exploitation des résultats. Bien souvent des approches multiples, plus ou moins simplifiées, restent nécessaires en complément pour valider les modélisations complexes, et bien évaluer les mécanismes essentiels du comportement de l'ouvrage.

Mots-clés : géotechnique, modélisation numérique, éléments finis, tridimensionnels, lois de comportement, interaction sol-structure.

\section{The interest of finite elements method tridimensional calculations in geotechnical design}

The paper aims to present an evaluation of the interest of 3D FEM calculations in geotechnical design. It reviews the obvious advantages of the method, but also some of its limitations. Then it shows some real case histories which show its potential but also its weaknesses. The conclusion is that in some case with simple but truly $3 \mathrm{D}$ geometry calculation can be considered as a standard way of design without any major difficulties. But in very complex and « extreme ») projects 3D calculations can help answer to very difficult questions, almost impossible to solve with simple methods, but with a very large amount of efforts and some simplifying hypothesis which must lead the engineer to be very careful when analyzing the results. Multiples approaches, including more or less simplified ones, are still necessary in order to validate the 3D models and to understand the fundamentals of the behavior. 


\section{Introduction}

Il est courant d'entendre dire que la modélisation numérique en géotechnique et, en particulier, la pratique des calculs aux éléments finis tendent à se banaliser. C'est déjà devenu pratiquement le cas pour les calculs bidimensionnels, mais les modélisations tridimensionnelles restent encore peu fréquentes dans les projets. Le but ce cet article est de discuter de l'intérêt des calculs éléments finis 3D dans les projets géotechniques.

Avant de poser, en 2012, la question de l'intérêt des calculs éléments finis tridimensionnels, il est utile de faire un petit retour en arrière, et de voir quelles ont été les évolutions récentes. Et tout d'abord rappelons ce qu'avaient écrit Magan et al. (1998) en introduction des Journées d'études sur la pratique des calculs tridimensionnels en géotechnique :

« ... l'application de méthodes de calcul complexes nécessite plus de paramètres que ceux que l'on a déterminés au cours du XX siècle... et, dans beaucoup de cas, le calcul tridimensionnel se retrouve tellement simplifié qu'il ne fait guère plus que le calcul bidimensionnel ou l'application d'abaques... L'avenir du calcul tridimensionnel dépend pour beaucoup des évolutions :

- du contenu des reconnaissances ;

- des types d'essais qu'il sera possible de réaliser ;

- de la représentativité des résultats de ces essais par rapport au comportement des terrains naturels ;

- de la description détaillée du déroulement des travaux de construction... »).

Avant d'évoquer la « validation de toutes les méthodes de calcul par rapport au comportement réel » et de conclure qu' (c un tel travail demandera de cinq à dix années $).$.

Plus probablement le calcul tridimensionnel restera réservé à la recherche et aux études d'ouvrages complexes et importants...

Il est donc temps, quatorze années plus tard, de voir comment les choses ont évolué, et comment la profession a su mettre à profit les différents outils devenus maintenant plus facilement accessibles.

\section{2}

\section{Quelques considérations générales}

Il convient d'abord de s'interroger sur la question elle-même : pourquoi mettre en cause l'intérêt des calculs tridimensionnels ? En effet, il apparaît évident qu'un certain nombre d'ouvrages relève manifestement de problèmes tridimensionnels, tels que :

- le cas élémentaire d'un pieu chargé horizontalement, qui en est une parfaite illustration élémentaire (Fig. 1) ;

- les cas de géologies très hétérogènes ;

- les ouvrages dont la géométrie ne peut pas se résumer à un coupe transversale unique, voire à plusieurs coupes (barrage-voûte ; cf. Fig. 2 ; intersections de tunnels, etc.) ;

- les cas d'ouvrages apportant des chargements et ayant des raideurs très hétérogènes, telles que par exemple les tours de grande hauteur avec noyau central (Fig. 3);
- les fondations isolées sur versant;

- les écoulements hydrauliques à partir ou vers des zones localisées, tels que des ouvrages-siphon ponctuels sous des tranchées couvertes recoupant la nappe phréatique ;

- le comportement des fronts de taille de tunnels ;

- les mécanismes d'interaction sol-structure entre éléments de renforcement linéaires (clous, inclusions, etc.) et le sol...

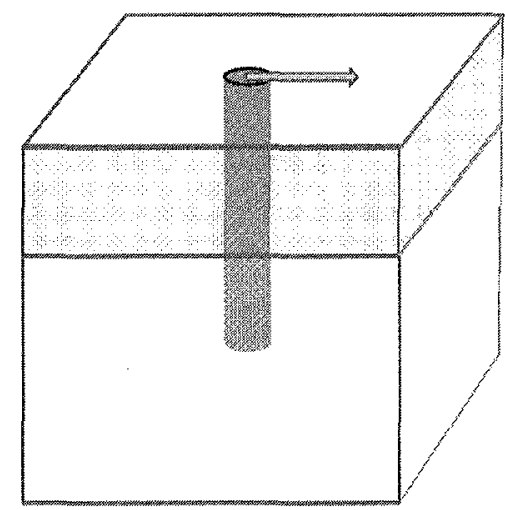

- Fe: Case of a horizontally loaded pile.

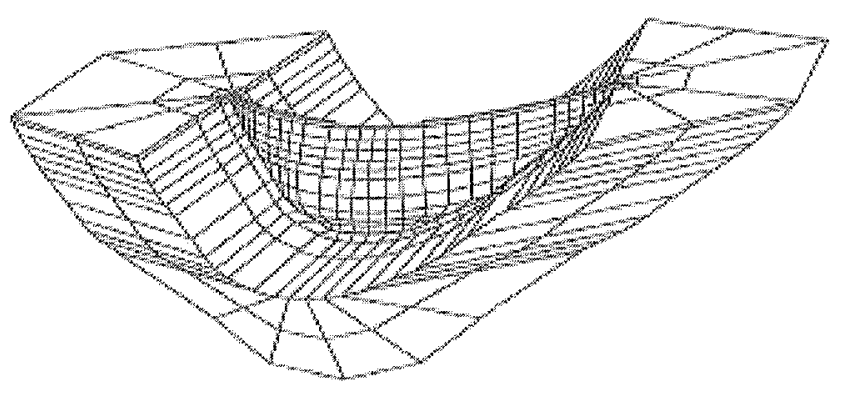

M.

Exemple de modèle 3D d'un barrage-voute
et sa fondation.
Example of a 3D model for a vault dam and its
foundations.

Mais il y a également un certain nombre de raisons pour lesquelles ce type de modélisations 3D n'est pas encore aussi fréquent dans la pratique que ce que pourrait suggérer l'énumération précédente des nombreux cas relevant de modèles 3D. Ces raisons sont pour l'essentiel liées à la modélisation elle-même du projet :

- la construction du modèle numérique peut être parfois très lourde, surtout lorsqu'on souhaite modéliser à la fois des sols et des éléments structurels (ouvrages, renforcements, fondations profondes...), et prendre en compte des conditions d'interface ;

- pour un projet complexe, le nombre de phases de calculs peut être très élevé (plusieurs dizaines), ce qui multiplie les risques d'erreurs, et rend toute modification de projet très difficile à gérer ;

- pour les mêmes raisons l'exploitation des résultats devient vite complexe, rendant plus délicate la validation du modèle ; 

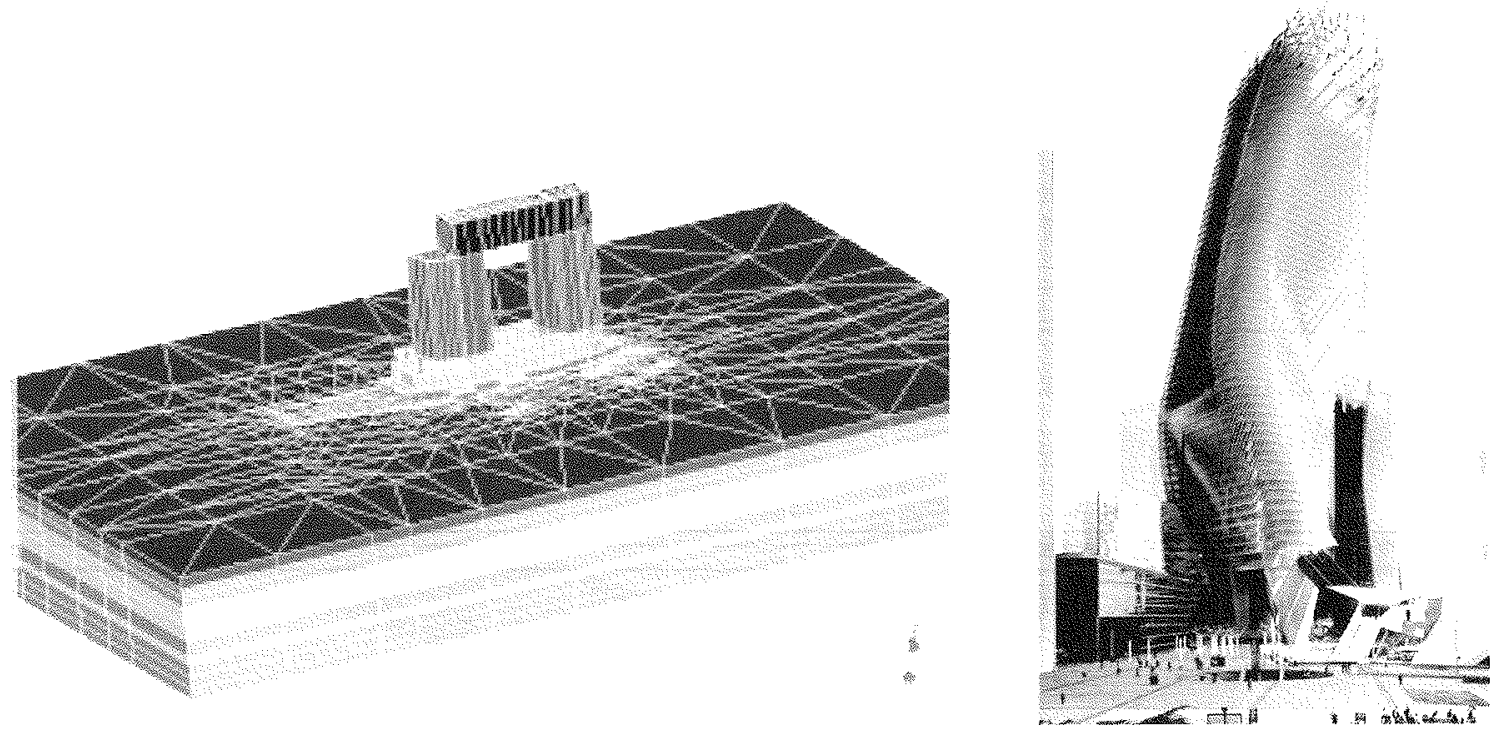

FG, 3 Exemple de modèle d'une tour complexe avec noyau central.

Example of a complex high rise tower model with central core.

- enfin, avec de tels modèles lourds, on ne peut pas facilement utiliser des lois de comportement des sols un peu élaborées : il faut souvent faire un choix entre soit un modèle détaillé en ce qui concerne la géométrie des horizons de sols et des ouvrages mais avec des lois de comportement ( simples ») de type élasticité ou élastoplastique en élasticité linéaire et critère de Mohr-Coulomb, soit un modèle simplifié par rapport à la réalité mais avec des lois de comportement plus complexes.

C'est pourquoi on cherche souvent à simplifier des problèmes 3D, par des modélisations axisymétricues, si la géométrie du problème le permet, ou en (c croisant ») des calculs 2D dans différentes directions, ou encore par des approches spécifiques telles que la méthode convergence - confinement dans les calculs de tunnels consistant en fait à introduire un (c chargement fictif ) tenant compte des phénomènes tridimensionnels qui se produisent au voisinage du front de taille.

Mais bien sûr de telles simplifications ne permettent pas toujours de répondre à la question posée et, en outre, il est souvent difficile d'apprécier l'impact des simplifications considérées sur les résultats obtenus.

Nous nous proposons d'illustrer ces apports et ces limites des calculs 3D par quelques exemples concrets de projets variés en terme de complexité des ouvrages ou de la géologie.
3

\section{Exemples de projets géotechniques}

\section{six}

\section{Plates-formes de tramway}

Les spécificités pour la modélisation de platesformes de tramway résident dans le caractère ponctuel des charges appliquées modélisant le passage des véhicules, dans un " problème géométriquement simple ) avec le plus souvent deux couches horizontales de chaussée ( béton ), mais avec des joints (Fig. 4). L'objectif d'un calcul élaboré est d'optimiser le dimensionnement, sachant que sur de grands linéaires, le gain de quelques centimètres sur l'épaisseur des chaussées peut être une source d'économie important.

Dans un tel cas, le calcul 3D permet de vérifier que les tassements sont assez uniformes dans la zone chargée, mais surtout de déterminer les contraintes de traction à la base de chaque sous-couche, en tenant compte de la présence du joint, que l'on peut comparer à la résistance en traction admissible du matériau, permettant ainsi d'ajuster les épaisseurs de couches (Fig. 5)

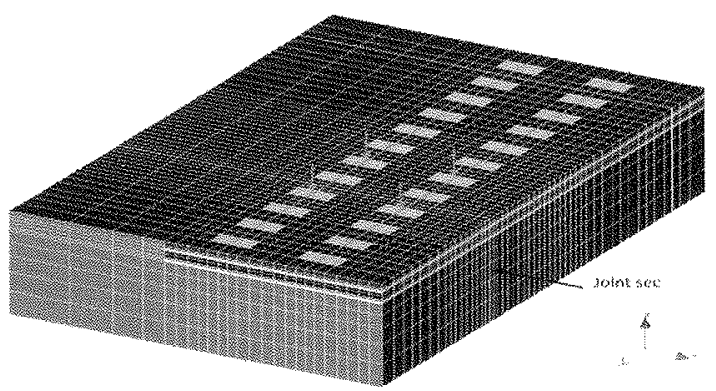




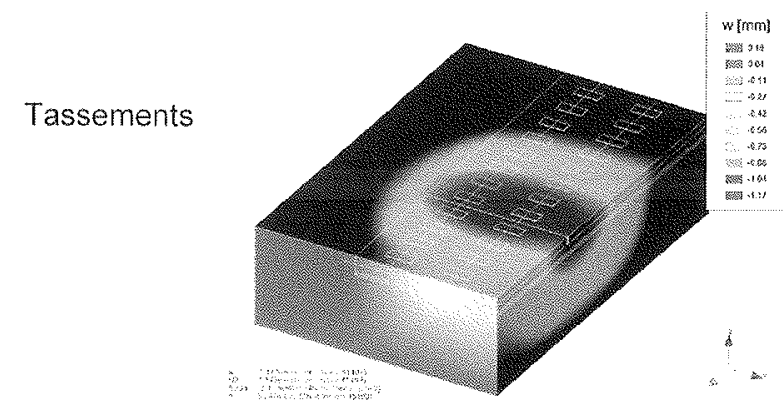

Contraintes de traction à la base

de chaque sous-couche

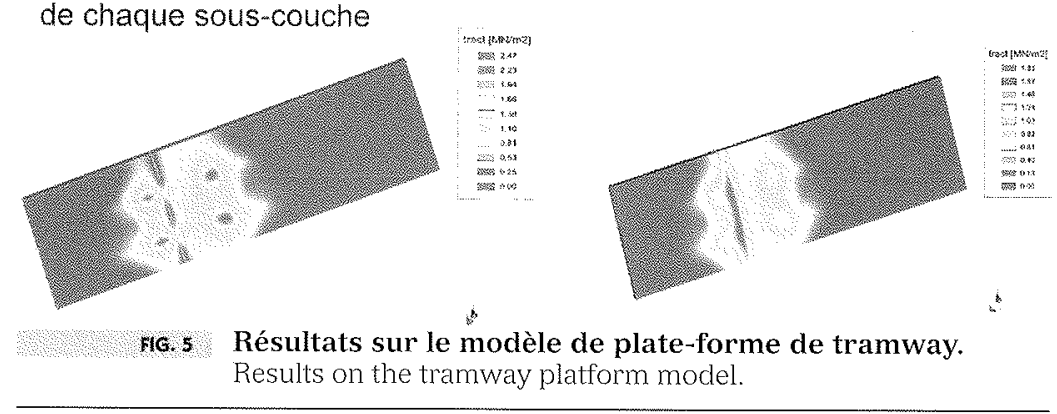

\section{6]}

\section{Excavation de faible longueur par rapport à sa largeur}

Une question qui se pose dans le cas d'une excavation de longueur limitée par rapport à sa largeur est celui de l'évaluation des soulèvements en fond d'excavation et en particulier des ( effets de bords ») du fait de la faible longueur. C'est clairement un cas où un modèle 2D ne peut répondre à la question, puisqu'il suppose implicitement une longueur d'excavation infinie.

L'exemple illustrant ce sujet concerne l'extension d'une station existante de métro de Toulouse, faisant $55 \mathrm{~m}$ de longueur et que l'on souhaite allonger à $70 \mathrm{~m}$, soit une extension de $15 \mathrm{~m}$, et de son incidence sur le tunnel existant que la nouvelle excavation va venir dégager (Le Bissonnais et Bretelle, 2002).

En pratique, et pour bien identifier les mécanismes, nous avons conduit trois modèles de calcul, un modèle 2D représentant le cas extrême d'une station de très grande longueur, et deux modèles 3D, l'un dans la situation actuelle avec la station de $55 \mathrm{~m}$ et l'autre après extension de $15 \mathrm{~m}$ (Fig. 6). En outre nous avons utilisé deux lois de comportement : une loi élastique linéaire avec plasticité selon le critère de Mohr-Coulomb, et une loi non linéaire avec le modèle Hardening Soil (élasticité non linéaire et écrouissage) en considérant pour cette loi un module $\mathrm{E}_{50 \mathrm{HS}}=1.1 \mathrm{E}_{\mathrm{MC}}\left(\mathrm{E}_{\mathrm{MC}}\right.$ étant le module utilisé dans le modèle Mohr-Coulomb) et un module en déchargement $\mathrm{E}_{u \mathrm{r}}=3^{*} \mathrm{E}_{50 \mathrm{H} \mathrm{SS}^{*}}$

Les résultats, représentés sur la figure 7, montrent que :

- l'effet géométrique 3D divise par 4 les soulèvements entre un modèle 2D et le modèle $3 \mathrm{D}$ ( réel ) avec extension de $15 \mathrm{~m}$, confirmant ainsi le rôle majeur des effets de bord ;

- l'effet d'une loi de comportement de type Hardening Soil, modélisant mieux les comportements en déchargement, réduit aussi par 4 les soulèvements entre un modèle linéaire et un modèle non linéaire.

Au final, il apparaît qu'une (c bonne modélisation », incluant à la fois les effets géométriques et un comportement réaliste en déchargement, réduit par un facteur 17 le soulèvement, qui passe de $50 \mathrm{~mm}$ pour le modèle 2D le plus simple à $3 \mathrm{~mm}$ pour le modèle $3 \mathrm{D}$ conforme à la géométrie réelle et avec une loi de comportement des sols plus pertinente!

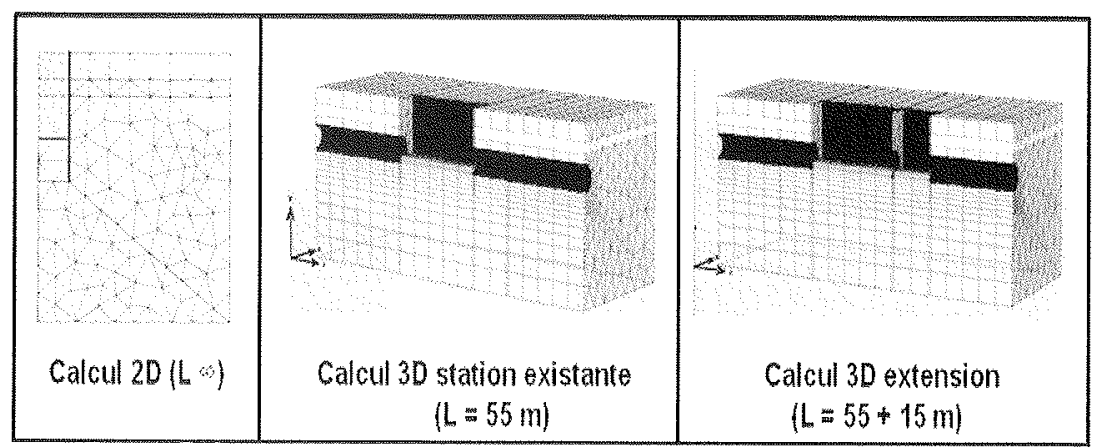




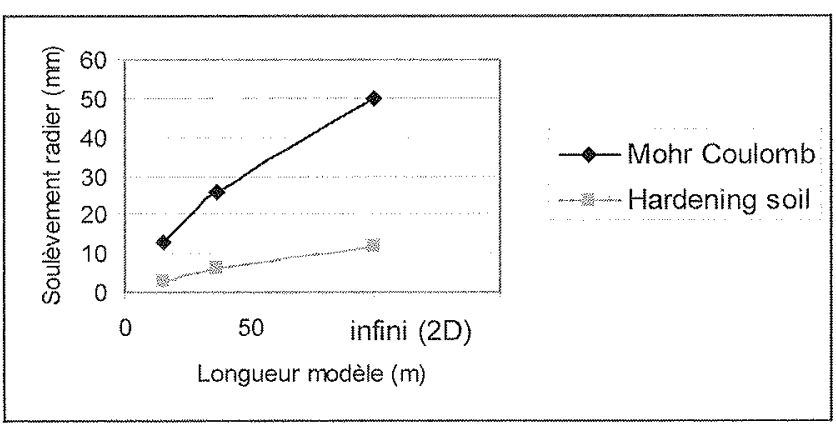

FIG. 1 Cas d'une excavation : effet du type de modèle et de la loi de comportement sur le soulèvement du fond.

Case of an excavation : effect of the type of model and constitutive law on the heave of the excavation bottom

\section{Grande tour à La Défense}

Le cas des grandes tours est un autre exemple où la modélisation 3D peut apporter des réponses utiles pour la conception d'un projet, lorsqu'on cumule des hétérogénéités de structure et de géologie.

Le cas de la tour Phare à La Défense (Guilloux, 2010), haute de $300 \mathrm{~m}$ illustre bien cette situation, avec un ouvrage à noyau central très rigide et des voiles façades supportant les planchers plus légers et ( souples », et également des appuis extérieurs ponctuels mais fortement chargés, le tout, bien sûr, dans un souci de compatibilité des déformations. La tour est fondée sur un radier général, apportant une contrainte moyenne de $1 \mathrm{MPa}$ et reposant sur la couche raide de calcaire grossier, surmontant elle-même des horizons plus compressibles d'argiles plastiques.

La question posée était essentiellement de savoir si ( l'effet dalle ») de l'horizon de calcaire grossier permettait de répartir suffisamment les charges en profondeur pour conduire à des tassements relativement uniformes, et quel était l'incidence de ces tassements sur les constructions voisines existantes (bâtiment, voies ferrées, ouvrages...).

La géométrie du modèle 3D a été détaillée au niveau de la structure pour éviter des simplifications excessives de type fondation souple ou au contraire infiniment rigide (Fig. 8). En revanche la modélisation du comportement du sol a été faite avec des lois ( simples » et notamment un comportement linéaire en phase élastique, mais en faisant varier le module au cours des étapes de calcul modélisant les phases de construction successives (déchargement lors de l'excavation, court terme, long terme...), et un critère de

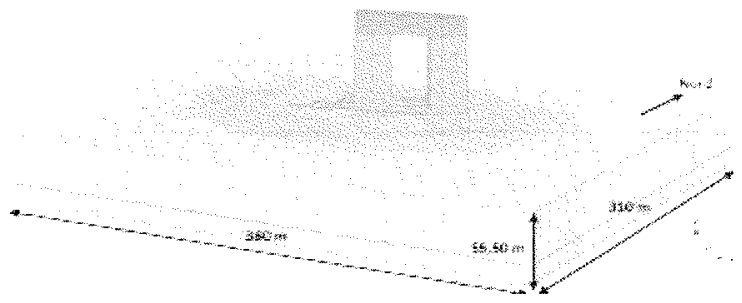

FG. 8 Modélisation de la tour Phare à La Défense. Model of the "Phare tower" ) in La Défense (Paris). plasticité de Mohr-Coulomb, intervenant peu en pratique, le comportement global restant principalement dans le domaine élastique.

Les résultats ont montré que, malgré des contraintes très variables sous les fondations, entre 0,4 et $3 \mathrm{MPa}$, le tassement global avait bien la forme d'une cuvette, légèrement excentrée du côté du noyau central, plus chargé, mais sans distorsions majeures (Fig. 9). De même ces calculs ont permis d'évaluer les tassements attendus sur les avoisinants, et également de définir une " carte des raideurs » à introduire dans les modèles de structures, et qui se sont avérés très fortement variables selon la position : en moyenne de l'ordre de $3 \mathrm{MN} / \mathrm{m}^{3}$ pour les zones les « moins chargées ») du radier à plus de $10 \mathrm{MN} / \mathrm{m}^{3}$ pour les zones les plus chargées et notamment les appuis ponctuels.

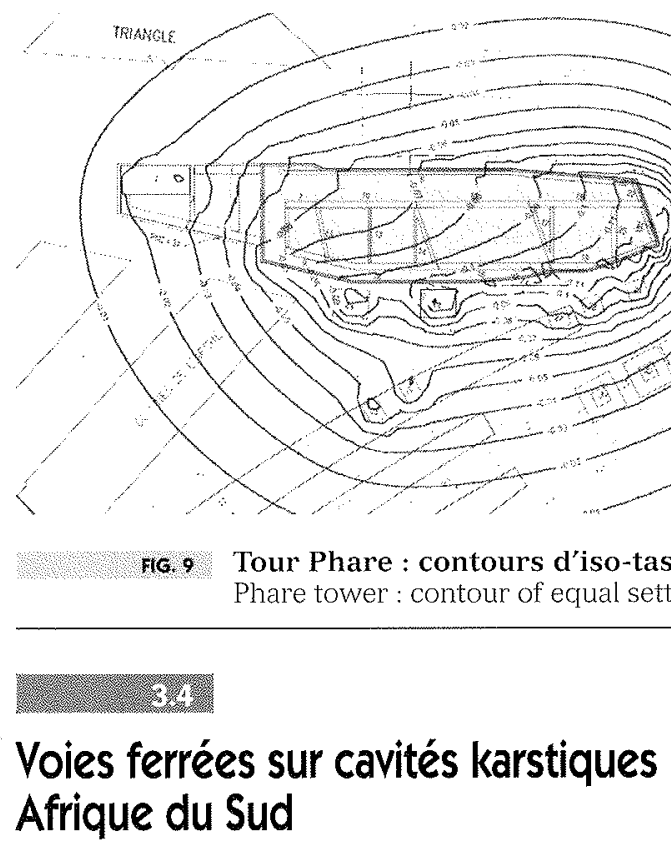

Cet autre exemple représente un cas un peu extrême, puiscu'il s'agit de dimensionner des structures en béton précontraint de $180 \mathrm{~m}$ de longueur permettant le franchissement de fontis de $15 \mathrm{~m}$ de diamètre (valeur de calcul retenue suite à une analyse statistique) d'une voie ferrée sur le projet Gautrain en Afrique du Sud (Bergère et al,, 2008; Bounatirou et al., 2009).

La question était double : quel est le comportement en déformation d'une telle structure ( pontant » un vide de $15 \mathrm{~m}$, ou plus précisément comment prendre en compte les effets de bords dans le calcul structurel (réduction des raideurs des appuis), mais surtout que se passe-t-il lorsque la poutre en $U$ repose sur un remblai de hauteur pouvant atteindre une dizaine de mètres lorsque le fontis de $15 \mathrm{~m}$ débouche à la base du remblai ? Quel est l'effet d'épanouissement de la cavité dans le remblai, en fonction des ses caractéristiques propres?

Après avoir mis au point une procédure numérique spécifique permettant d'éliminer les éléments « tombant dans la cavité ») les calculs ont permis de mieux comprendre les mécanismes de décollement entre la poutre et son sol support (Fig. 10), et les effets d'entonnoir dans le remblai, pour une cavité cylindrique placée à différentes distances de l'axe de la structure linéaire, et en zone courante de poutre ou au droit d'un joint (Fig. 11). 

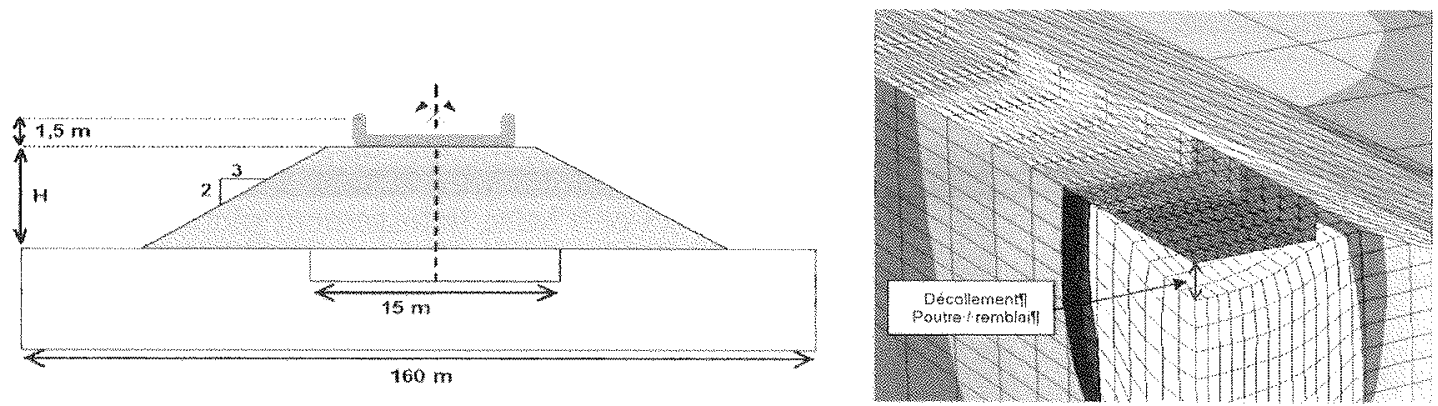

FG. 10 Modélisation d'une poutre reposant sur un remblai au-dessus d'une cavité.

Model of a beam resting on an embankment over a cavity.

Les résultats ont ainsi, grâce à des études paramétriques, fourni des éléments permettant de trouver le compromis entre des solutions de traitement aux liants hydrauliques du remblai, ou de renforcement de ce remblai par géogrilles destinées à limiter l'effet entonnoir, et un surdimensionnement de la structure pour franchir une brèche de $15 \mathrm{~m}$ à la base du remblai, mais pouvant atteindre 20 à $25 \mathrm{~m}$ sous la poutre. Ils ont également fourni des éléments de raideur pour le dimensionnement de la structure.

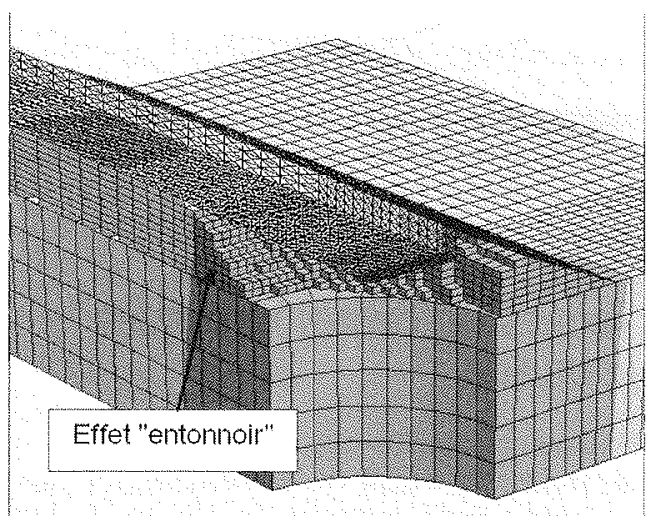

Fic 11 Modélisation de l'effet-entonnoir dans le remblai au-dessus de la cavité

Modelisation of the funnel effect in the embankment above the cavity.

\section{5}

\section{Grande excavation à Monaco}

L'exemple de la tour Odéon à Monaco est également un cas extrême, puisqu'il s'agit d'une excavation de $70 \mathrm{~m}$ de profondeur, constituée de parois berlinoises et parois moulées superposées, ancrées par tirants précontraints pour la partie haute, et soutenues par les planchers des sous-sols pour la partie basse, avec une méthode de construction Up \& Down (Fig. 12). L'ensemble est situé sur un versant à forte pente, au milieu de constructions existantes dont les déformations devaient être strictement contrôlées.

Il a fallu bâtir un modèle incluant plus de 500 tirants, pieux et micropieux, et comportant au final 695000 élé ments avec 57 phases de calcul! L'ensemble a nécessité plusieurs mois de travail, mais les résultats ont permis d'estimer que les tassements et déplacement horizontaux des avoisinants ne dépassaient pas 5 à $10 \mathrm{~mm}$, et plus généralement de fournir des valeurs de référence pour l'application de la méthode observationnelle mise en œuvre sur ce chantier.

Elle a aussi permis de quantifier les effets de cette excavation de faible largeur au sein d'un versant raide, d'une part par effets de voûte contournant l'excavation et venant " surcharger » les « culées de ce barragevoûte », mais aussi vis-à-vis de la stabilité générale du versant en cours et après construction (par une procédure de type $\mathrm{c}-\varphi$ réduction), impossible à analyser correctement dans des analyses classiques bidimensionnelles.
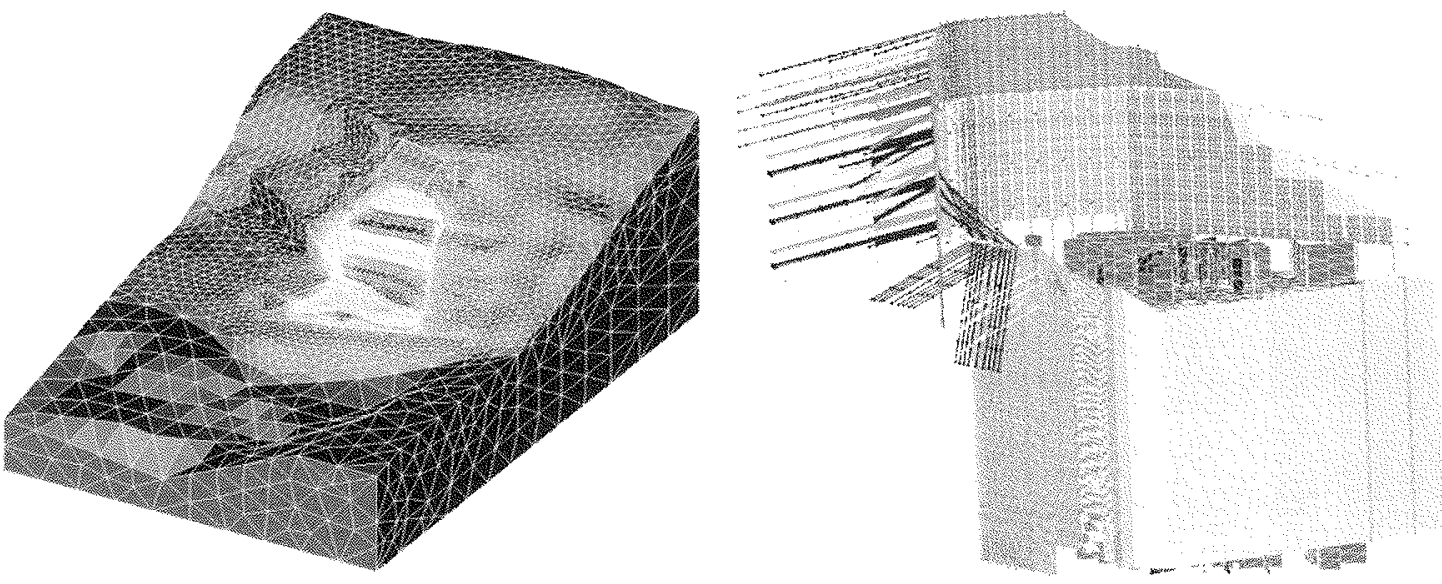

ra. 12 Tour Odéon à Monaco : vue générale du modèle et vue des éléments structuraux. Odeon tower in Monaco : general view of the model and view of the structural elements. 


\section{Bâtiment complexe : la Fondation Louis Vuitton pour la Création}

Le dernier exemple présenté est celui de la Fondation Louis Vuitton pour la Création, en cours de construction au jardin d'Acclimatation à Paris (Beaussier et al., 2011) ; c'est un bâtiment prestigieux, conçu par l'architecte Franck Gehry. Il est construit à l'intérieur d'une excavation réalisée en parois moulées, et est fondé sur un radier de $2,6 \mathrm{~m}$ d'épaisseur et $40 \mathrm{~m}$ de largeur environ; la géométrie de l'ouvrage est complexe (Fig. 13), avec des descentes de charges fortement dissymétriques et une structure très sensible aux mouvements différentiels; en outre il fonctionne ( en déchargement) (poids des terrains excavés en moyenne supérieur aux charges apportées) dans un contexte géologique présentant en profondeur des couches argileuses au comportement différé marqué.

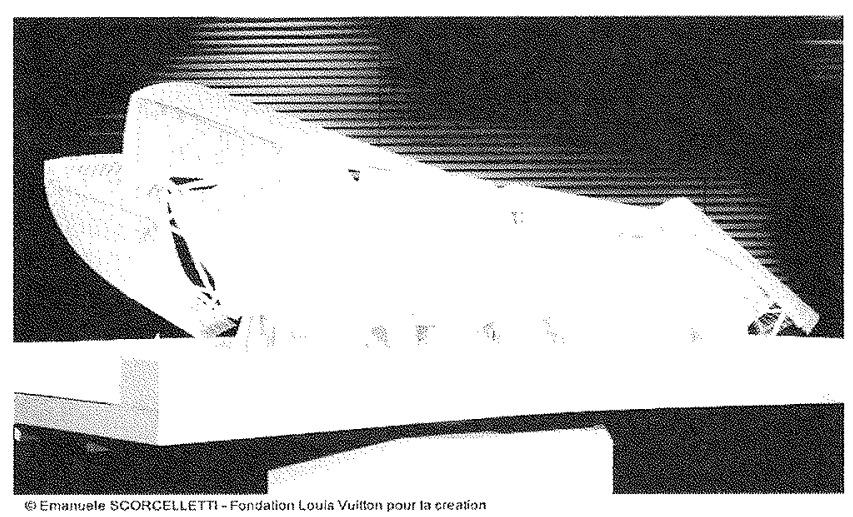

NG. 13 Fondation Louis Vuitton pour la Culture : vue de l'ouvrage.

View of the "Fondation Louis Vuitton pour la Culture » museum.

Il a fait l'objet de deux approches complémentaires : - au stade du projet, une modélisation 3D privilégiant l'analyse de l'interaction sol-structure par le biais d'un modèle éléments finis 3D détaillé sur la structure (notamment du radier et des voiles d'infrastructures) (Fig. 14), dont la lourdeur a conduit à utiliser des lois de comportement simples (élasticité linéaire, critère de plasticité de Mohr-Coulomb), avec bien sûr un choix approprié de paramètres de calculs et des analyses complémentaires sur la consolidation ;

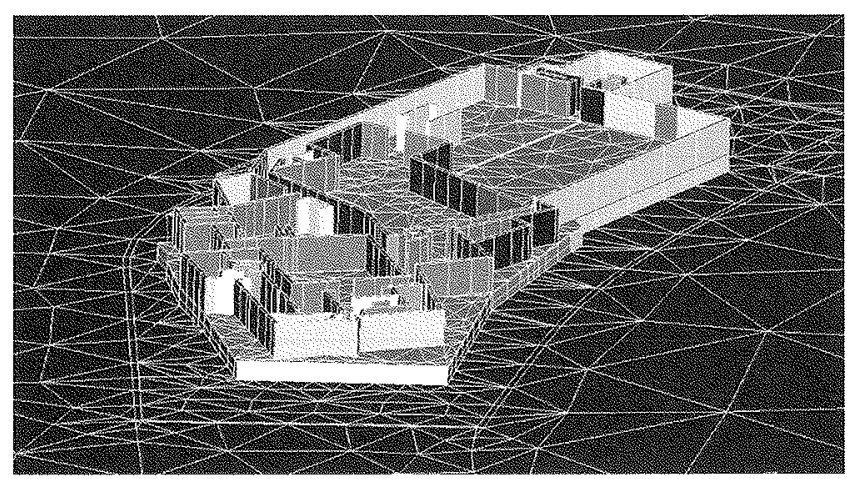

Fic 14 Vue du modèle de Fondation Louis Vuitton pour la Culture.

View of the model for the « Fondation Louis Vuitton pour la Culture s.
- au stade de l'exécution, à l'aide de plusieurs modélisations 2D, donc avec une modélisation simplifiée de la structure et des charges appliquées, mais permettant d'utiliser des lois plus évoluées de comportement des sols, prenant en compte l'écrouissage et les phénomènes de consolidation, et de réaliser des études paramétriques sur les variations probables des paramètres géotechniques.

En outre les paramètres géotechniques utilisés pour les calculs 2D ont pu être calés sur les essais géotechniques réalisés lors des reconnaissances de projet, mais aussi grâce à une rétro-analyse des mouvements enregistrés sur chantier lors des phases d'excavation.

Les calculs 3D ont montré que, grâce à la forte épaisseur du radier, les contraintes sous le radier étaient relativement uniformes, ce qui a permis de valider le principe d'une approche bidimensionnelle.

Dans l'ensemble les résultats des deux approches sont comparables et cohérentes avec le comportement réel mis en évidence par les auscultations. L'avancement du chantier a permis de faire une comparaison plus précise entre les soulèvements de fond de fouille calculés par les deux modèles et ceux mesurés sur chantier sur une période de trois mois entre la fin d'excavation et le coulage du radier. Le modèle 3D prévoyait un soulèvement de $1,3 \mathrm{~mm} / \mathrm{mois}$, le modèle 2D de $0,7 \mathrm{~mm} / \mathrm{mois}$, et la réalité montre en moyenne $1 \mathrm{~mm} / \mathrm{mois}$.

La figure 15 montre que, après coulage du radier, les deux modélisations conduisent à des cuvettes de tassements de formes comparables, mais avec $14 \mathrm{~mm}$ de tassement maximal dans le calcul 3D contre $10 \mathrm{~mm}$ dans les calculs 2D. Et la réalité est très proche des calculs 2D.

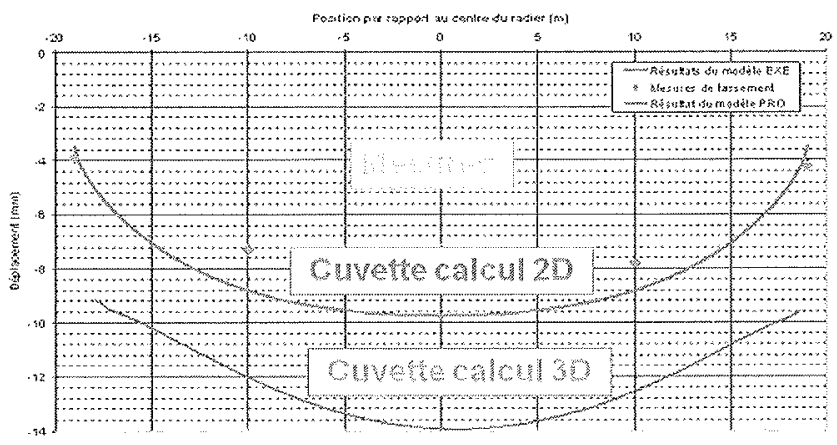

न6. 15 Tassements enfin de bétonnage du radier. Settlements at the end of the raft concreting phase.

Dans ces deux phases, l'hypothèse de déformation plane est justifiée puisque les chargements sont uniformes, et les différences entre modèles ne sont dues qu'au choix de lois de comportement différentes et des paramètres associés.

C'est lors de phases suivantes que ces différences seront plus importantes, du fait des dissymétries de chargement, comme l'illustre la figure 16, où l'on voit que le modèle 2D conduit à des tassements très uniformes sur toute la largeur du radier, tandis que le calcul 3D montre une dissymétrie assez marquée entre les côtés les plus chargés et les plus légers, avec des différences de tassements de $6 \mathrm{~mm}$ à court terme, et $9 \mathrm{~mm}$ à long terme. 


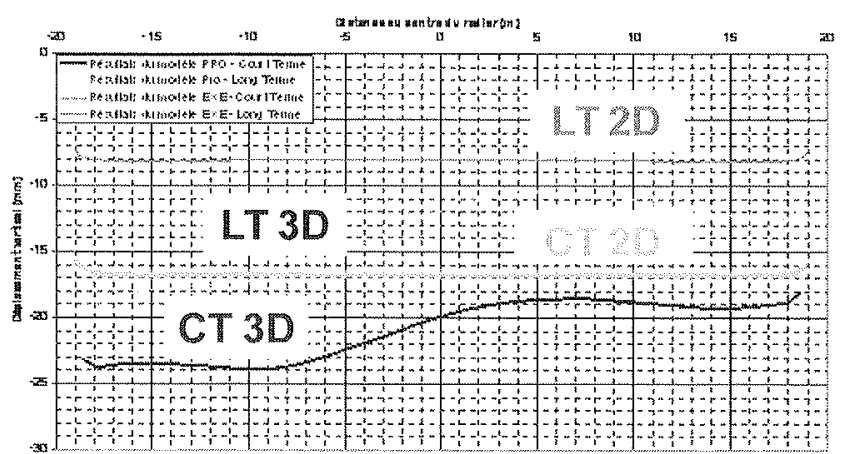

FG. 16. Tassements calculés en fin de construction Calculated settlements at the end of construction.

Ce cas illustre bien la complémentarité des deux modèles, le modèle $3 \mathrm{D}$ permettant de valider les simplifications à introduire dans des calculs 2D, lesquels semblent être plus proches de la réalité en ce qui concerne l'amplitude des tassements, mais « gommer s) un peu trop fortement l'effet des charges non uniformes.

\section{4}

\section{Synthèse et conclusions}

Comme souvent dans ce genre de problématique, il n'y a pas de réponse dogmatique. Les modélisations tridimensionnelles, qui sont devenues assez facilement ( accessibles ) même en dehors des organismes de recherche, trouvent leur place dans la panoplie des outils à la disposition de l'ingénieur. Mais elles ne doivent pas être considérées comme « l'outil absolu » permettant de répondre à tous les problèmes.

Leur utilisation ne pose guère de difficultés lorsqu'il s'agit de mettre en ouvre des modèles simples avec un caractère tridimensionnel très marqué, comme les cas des plates-formes de tramways ou d'excavations de longueur limitée que nous avons présentées dans cet article, contredisant quelque peu la prédiction évoquée en introduction « le calcul tridimensionnel restera réservé à la recherche et aux études d'ouvrages complexes et importants ).

Mais il est vrai que la tendance est forte à l'appliquer à des cas beaucoup plus complexes en termes de géométrie des terrains et des ouvrages, de comportements géotechniques spécifiques, et d'interactions solstructure multiples. De telles modélisations ne peuvent être abordées qu'au prix d'efforts importants, en particulier sur les délais pas toujours compatibles avec les contraintes de planning d'un projet, et moyennant des choix de simplifications préalables, privilégiant soit la structure, soit le sol, mais plus difficilement les deux ensembles.

Et gardons à l'esprit que, dans ces cas extrêmement complexes, une modélisation lourde ne permet pas toujours d'identifier facilement les mécanismes de comportement global de l'ouvrage, et qu'il y a tout intérêt à conduire en parallèle des approches analytiques simples ou des modélisations 2D, qui permettent de mieux appréhender les phénomènes, et de valider les simplifications que l'on devra inévitablement introduire dans les calculs complexes.

\section{Bibliographie}

Beaussier A., Guilloux A., Verschuere J.

- La Fondation Louis Vuitton pour la

Création : modélisations géotechniques croisées 2D et 3D et confrontation avec les auscultations. Revue française de géotechnique, $\mathrm{n}^{\circ}$ 137, 2011.

Bergère A., Guilloux A., Chapron G. Modélisation de la remontée d'un fontis à travers un remblai. Travaux $n^{\circ} 856$, octobre 2008
Guilloux A., Simon B. - La problématique des fondations des grandes tours; le cas de La Défense à Paris. Présenté à la conférence franco-maghrébine en ingénierie géotechnique. Tunis, 9-11décembre 2010

Le Bissonnais H., Bretelle S. - Analyse de soulèvements induits par l'agrandissement d'une grande excavation. Comptes rendus du $3^{\circ}$ symposium international TC 28, Toulouse. Éditions spécifiques, 2002.
Magnan J.-P., Guilloux A., Mestat Ph. - La pratique des calculs tridimensionnels en géotechnique. Presses des Ponts, Journées d'études, Paris, 24-25 novembre 1998.

Bounatirou S., Storry R., Viallon J.-P., Pignerol F, Demonceaux T., Guilloux A., Bergère A. - Le projet Gautrain : la zone dolomitique. Travaux, $n^{\circ}$ 858, février 2009. 